\title{
THE FUTURE CONDITIONAL
}





\section{THE FUTURE CONDITIONAL \\ Building an English-Speaking Society in Northeast China}

Eric S. Henry 


\section{Copyright ( 2021 by Cornell University}

All rights reserved. Except for brief quotations in a review, this book, or parts thereof, must not be reproduced in any form without permission in writing from the publisher. For information, address Cornell University Press, Sage House, 512 East State Street, Ithaca, New York 14850. Visit our website at cornellpress.cornell.edu.

First published 2021 by Cornell University Press

\section{Library of Congress Cataloging-in-Publication Data}

Names: Henry, Eric S., author.

Title: The future conditional : building an English-speaking society in northeast China / Eric S. Henry.

Description: Ithaca [New York] : Cornell University Press, 2021. | Includes bibliographical references and index.

Identifiers: LCCN 2020034865 (print) | LCCN 2020034866 (ebook) | ISBN 9781501754906 (hardcover) | ISBN 9781501755163 (paperback) | ISBN 9781501754913 (epub) | ISBN 9781501754920 (pdf)

Subjects: LCSH: English language-Social aspects—China. | English languageStudy and teaching-Social aspects—China. | English language-Study and teaching—Chinese speakers. | Group identity—China.

Classification: LCC P40.5.G762 C45 2021 (print) | LCC P40.5.G762 (ebook) | DDC $428.0071 / 051-\mathrm{dc} 23$

LC record available at https://lccn.loc.gov/2020034865

LC ebook record available at https://lccn.loc.gov/2020034866

Cover photograph by the author. 
For Jing, Layton, Riley, and Tai 
